\title{
Détection CPG/SM des biomarqueurs spécifiques de l'élevage des vins en barriques
}

\author{
N. Vivas ${ }^{1, *}$, G. Bourgeois ${ }^{3}$, Y. Glories ${ }^{2}$, M. Augustin ${ }^{2}$ et C. Vitry ${ }^{3}$ \\ ${ }^{1}$ Tonnellerie Demptos, détaché à la Faculté d'Enologie, Université Victor Segalen Bordeaux II, \\ 351 cours de la libération, 33405 Talence, France \\ ${ }^{2}$ Laboratoire de Chimie Appliquée, Faculté d'Enologie, Université Victor Segalen Bordeaux II, France \\ ${ }^{3}$ Centre d'Études Structurales et d'Analyse des Molécules Organiques, CNRS URA 35, \\ Université Bordeaux I, 351 cours de la libération, 33405 Talence, France
}

\begin{abstract}
Proceeding with a similar way as previous works, we characterised the presence of lignins in wines. This natural polymer was chosen as biomarker. It's a remarkably stable in time and typical wood component. We used the thioacidolysis reaction with ethanethiol and boron trifluoroetherate as reagents, to obtain some characteristic phenylpropane units from the lignin polymer. Identification was performed by mass spectrometry coupled with gas chromatography (GPG/SM) of thioacidolysis products in trimethylsililated form. The aim of this study is the demonstration of wine aging in barrel before bottling.
\end{abstract}

Key words. Ancient wine - barrel aging - lignin - thioacidolysis.

La barrique en bois constitue un très ancien récipient destiné à la fois à la vinification, à l'élevage, au transport et à la conservation des vins [10]. Mais le bois n'est pas un matériau inerte: il favorise d'abord les échanges gazeux entre le vin et l'atmosphère environnante et permet ensuite la dissolution de nombreux constituants sapides et odorants qui participent aux caractères organoleptiques des vins (Vivas et al. 1991 ; [12]). C'est essentiellement de la durée du séjour en barrique que dépend l'intensité de ces diverses réactions. Pour de longues périodes, le vin est à ce point modifié que sa composition et sa qualité sont radicalement différentes de celles du départ.

Sur le plan de l'histoire, à partir du $1^{\text {er }}$ siècle après J.-C, le tonneau commence à s'imposer pour la conservation des vins. Jusqu'alors, il était pour l'essentiel destiné à la bière de la civilisation celtique. Face à elle, la civilisation grécoromaine rayonnante avait imposé partout l'amphore («amphoreus» qui signifie en grec qu'on porte par deux anses). C'est le déclin de l'empire romain qui a permis aux Gaulois de constater par eux-mêmes les avantages et les inconvénients respectifs de ces deux récipients. Le tonneau moins fragile, plus facile à manipuler et à empiler, s'est très facilement imposé. Par le passé, le principal rôle du tonneau était marchand, il constituait alors le moyen de transporter les vins par bateaux. Au fil des siècles, les amateurs de vin finirent par découvrir que pendant le transport, pour peu qu'aucune altération ne vienne gâcher la qualité du vin, il se produisait un véritable affinage à l'origine de la notion actuelle «d'élevage ». Mais lorsque les tonneaux se vidaient au fur et à mesure de la consommation du vin, le produit se dépréciait rapidement et devenait vite «acétique» et impropre à la consommation. C'est à partir du XVII ${ }^{\mathrm{e}}$ siècle qu'apparut la solution: la mise en bouteille. L'élevage des vins est devenu alors l'affaire des producteurs qui ont pris, très progressivement, au fil des siècles, pour habitude, d'intégrer au sein de «l'art et la manière de faire le vin » l'étape d'élevage en barriques. Il est donc intéressant, pour retracer l'histoire des pratiques œnologiques au fil du temps**, de repérer dans les vins, l'indice de l'utilisation éventuelle de la barrique.

\section{Recherche de biomarqueurs spécifiques}

Les marqueurs servant à déterminer l'origine et l'histoire d'un vin, doivent être stables sur le plan de leur structure native, surtout lorsqu'il s'agit d'un vin très âgé et conservé dans des conditions difficiles.

Certaines substances ont été mentionnées dans la littérature pour servir de marqueurs: ainsi [9] proposèrent le dosage d'une coumarine, la scopolétine, comme marqueur de l'élevage en barrique; malheureusement, il s'avère que les coumarines sont progressivement dégradées au cours du temps et disparaissent après quelques dizaines d'années ; elles sont donc inutilisables dans le cas qui nous intéresse.

Les polymères du bois sont des marqueurs beaucoup plus stables que les coumarines et s'avèrent beaucoup plus fiables: les polysaccharides (polymères osidiques), les lignines et les formes polymérisées des ellagitanins (polymères phénoliques). Afin de déterminer la nature de ces polymères, on peut mettre en œuvre une méthode de dépolymérisation en milieu acide, puis analyser les unités élémentaires libérées caractéristiques de ces macromolécules.

Les polymères présents dans les bois, susceptibles d'être utilisés comme marqueurs, sont de trois sortes :

- les polysaccharides du bois : ils n'ont malheureusement pas une composition suffisamment différente de ceux

* Correspondance et tirés-à-part.

Reçu le 9 octobre 1997 ; révisé le 13 janvier 1998 ; accepté le 16 janvier 1998.

** À ce propos, nous suggérons d'utiliser le terme d'Archœnologie pour l'ensemble des disciplines qui s'intéressent aux vins d'origine ancienne. 
du vin et leurs utilisation comme marqueurs a été abandonnée ;

- les ellagitanins sont spécifiques de quelques espèces de bois (chênes européens, châtaignier, chêne blanc d'Amérique). Ces molécules sont cependant trop oxydables pour les retrouver dans des vins d'âge supérieur à 10 ans [13]. En revanche, l'acide ellagique représentant l'unité structurale élémentaire des ellagitanins est une molécule particulièrement stable dont on peut facilement détecter la présence ;

- la lignine semble être le marqueur le plus riche en informations sur la nature du récipient ayant contenu le vin. Ce polymère naturel, constitué d'unités d'alcools phénoliques hydroxylés (hydroxyphényles), monométhoxylés (gaïacyles) et diméthoxylés (syringyles), est typique de l'élevage en barriques. La lignine est stable dans le temps, peu sensible à l'hydrolyse dans les conditions de pH acide; de plus, les vins en sont naturellement dépourvus. L'aspect particulièrement stable de ce biomarqueur a été démontré [7] à l'occasion d'une étude sur les suspensions dans les eaux de rivière; la méthode leur a permis de déterminer la source végétale majoritaire à l'origine de la matière organique adsorbée sur les particules en suspension. Les lignines peuvent donc être utilisées comme marqueurs spécifiques de la conservation des vins en contact avec le bois; cette démarche a d'ailleurs déjà été employée [11] sur des cognacs élevés en barrique durant des périodes plus ou moins longues.

\section{Origine de l'échantillon}

Notre étude porte sur un échantillon de vin datant de la fin du $\mathrm{XIX}^{\mathrm{e}}$ siècle et ne constitue donc pas réellement un support archéologique stricto sensu. Cependant, l'intérêt du travail présenté est de mettre à la disposition des spécialistes de ce secteur scientifique une méthodologie adaptée au cas des vins anciens, permettant de retracer une partie de leurs techniques de production.

Le vin étudié provient de l'épave du Marie-Thérèse, un trois-mâts-barque de 503 tonneaux construit en 1865 à Bordeaux. Ce navire marchand a fait naufrage le 29 février 1872 au sud-ouest de l'île de Belitung située dans le détroit de Gaspar (séparant les îles de Sumatra et de Bornéo), alors qu'il transportait diverses marchandises à destination de Saigon. Dans l'inventaire des fouilles, réalisé le 5 octobre 1991, figuraient 2022 bouteilles de vin de Bordeaux, parmi lesquelles des Gruaud-Larose (Saint-Julien, Médoc) du millésime 1868. Ce vin se présente en bouteilles bouchées par du liège recouvert par de la cire. Seuls des échantillons issus de bouteilles intactes ont été retenus pour les analyses.

\section{Matériel et méthodes}

\section{Échantillon de lignine pure}

Nous utilisons, comme échantillons de référence, des fractions lignines extraites au dioxane (LD), dans les conditions décrites par Vivas et al. (1996). Des lignines LD provenant de Quercus robur et $Q$. petraea (chênes Européens habituellement utilisés), servent de témoin. A titre de comparaison nous avons purifié, dans les mêmes conditions, des lignines des espèces $Q$. alba (chêne blanc d'Amérique), Castanea sativa (châtaignier Européen) et $Q$. lanuginosa (chêne pubescent très répandu en France), Q. farnetto (chêne de l'Europe centrale) et $Q$. toza (chêne rencontré dans le nord de l'Espagne et en Italie).

\section{Isolement des lignines du vin}

Au cours du temps, il s'est produit une lente pénétration du sel marin, principalement du chlorure de sodium, au travers du bouchon. L'augmentation de la force ionique du milieu a provoqué la précipitation de la plus grande partie des composés phénoliques. Nous récoltons alors la fraction phénolique insoluble au fond de la bouteille. L'élimination des sels est ensuite réalisée par dialyse contre $40 \mathrm{~L}$ d'eau distillée désionisée. Le précipité dessalé est alors lyophilisé et $50 \mathrm{mg}$ sont utilisés pour déterminer la présence éventuelle de lignines.

\section{Analyse générale des vins}

Les analyses habituellement pratiquées sur les vins (alcool, sucres réducteurs, $\mathrm{pH}$, acidité totale, acidité volatile) sont réalisées selon les protocoles recommandés par l'OIV (Office International de la vigne et du Vin, 1977). Le dosage des chlorures et du sodium est pratiqué par absorption atomique [4]. Les composés phénoliques et la couleur des vins sont évalués par les méthodes classiques [1].

\section{Dosage de l'acide ellagique}

L'acide ellagique, dilactone de l'acide gallique, est dosé par «Chromatographie Liquide Haute Performance » (HPLC) en phase inverse, dans les conditions décrites par Vivas et al. (1996). Pour améliorer la détection de ce composé nous extrayons $100 \mathrm{~mL}$ de vin centrifugé (4000 tpm, $30 \mathrm{~min}$ ) par successivement 20,10 et $10 \mathrm{ml}$ d'éther diéthylique. Les phases organiques sont collectées puis l'éther est éliminé à l'évaporateur rotatif $\left(40^{\circ} \mathrm{C}\right)$. Le résidu est repris par $1 \mathrm{~mL}$ de méthanol et analysé par HPLC. L'identification du composé est permise par la comparaison du temps de rétention avec celui du composé de référence et d'après son spectre UV (maximum à 280 et $370 \mathrm{~nm}$ ). Une confirmation est donnée par la réalisation d'un spectre de masse en impact électronique $(70 \mathrm{eV})$ du résidu d'extraction.

\section{Thioacidolyse et analyse par couplage "Chromatographie en Phase Gazeuse/ Spectrométrie de Masse " CPG/SM) des produits formés, sous forme de dérivés triméthylsilylés}

La méthode appliquée est décrite dans le détail par Rolando et al. (1992). Elle avait été mise au point par Lapierre (1986). Brièvement, elle consiste en une thioacidolyse de la lignine (traitement au $\mathrm{BF}_{3}$ dans du dioxane/éthanethiol ; 8,75:1). Les produits de dégradation, se présentant sous forme de dérivés thioéthylés, sont analysés par CPG/SM après silylation (BSTFA/TMS). Dans le cas de cette étude, où les concentrations attendues en lignine sont faibles, nous avons amélioré le rendement de la réaction en augmentant la proportion de réactifs permettant la thioacidolyse quasi-complète des traces de lignines collectées. En accord avec nos travaux antérieurs [14], il apparaît que $15 \mathrm{~mL}$ de réactif pour $50 \mathrm{mg}$ de précipité correspond à la quantité optimale. L'analyse CPG/SM des produits de 
thioacidolyse est réalisée dans les conditions suivantes : colonne Chrompack CPSil5-CB ${ }^{\mathrm{TM}}$ (longueur $25 \mathrm{~m}$, diamètre $0,25 \mathrm{~mm}$ ), température de l'injecteur $240{ }^{\circ} \mathrm{C}$, température du four programmée de $120^{\circ} \mathrm{C}$ à $260{ }^{\circ} \mathrm{C}$ à raison de $4{ }^{\circ} \mathrm{C} / \mathrm{min}$ - spectromètre de masse Fisons Autospec-Q (conditions d'ionisation: $70 \mathrm{eV}, 150 \mu \mathrm{A}, 200{ }^{\circ} \mathrm{C}$ ).

\section{Résultats et discussion}

\section{Composition générale du vin}

Dans le tableau I nous avons regroupé les résultats concernant les analyses classiques des vins, les analyses de quelques éléments minéraux et les analyses des composés phénoliques, dont l'acide ellagique, marqueur d'un contact entre du bois et le vin. A titre de comparaison nous donnons la composition moyenne des vins du Médoc produits de nos jours.

Le degré alcoolique du vin de 1868 est faible mais en accord avec les niveaux rencontrés pour les vins de l'époque (9,5\% vol.). L'acidité totale et le $\mathrm{pH}$ indiquent que le vin à été produit avec des raisins particulièrement mûrs, car à l'époque, pour des raisons sanitaires, les raisins étaient récoltés plus précocement, avec pour conséquence des acidités totales nettement plus élevées. La disparition de l'acide malique et le faible taux de sucres réducteurs résiduel, sont les témoins d'une vinification bien maîtrisée avec un achèvement de la fermentation alcoolique et de la fermentation

Tableau I. Résultats des dosages classiques sur les vins, du dosage de quelques éléments minéraux et de l'analyses des composés phénoliques du raisin et du bois des barriques.

\begin{tabular}{lrr}
\hline Paramètres & \multicolumn{2}{c}{ Résultats de dosages } \\
& $1868 \dagger$ & $1995 \$$ \\
\hline
\end{tabular}

Analyses générales:

\begin{tabular}{|c|c|c|}
\hline Alcool(\% vol.) & 9,45 & 12 \\
\hline Sucres réducteurs $(\mathrm{g} / \mathrm{L})$ & 1,8 & $<2$ \\
\hline $\mathrm{pH}$ & 3,4 & 3,8 \\
\hline Acidité totale* & 3,9 & 3,5 \\
\hline Acidité volatile* & 0,92 & $<0,5$ \\
\hline Acide malique (g/L)\# & 0 & 0 \\
\hline \multicolumn{3}{|l|}{ Eléments minéraux: } \\
\hline Sodium (mg/L) & 490 & $\leq 25$ \\
\hline Potassium (mg/L) & 880 & $\leq 1000$ \\
\hline \multicolumn{3}{|l|}{ Composés phénoliques: } \\
\hline Phénols totaux§ & 40 & 50 \\
\hline Procyanidines $(\mathrm{g} / \mathrm{L})^{\circ}$ & 1,1 & 3,5 \\
\hline Acide ellagique $(\mathrm{mg} / \mathrm{L}) £$ & 3,8 & - \\
\hline Anthocyanes $(\mathrm{mg} / \mathrm{L})^{\circ}$ & $<30$ & 600 \\
\hline
\end{tabular}

malolactique, gages d'une bonne et longue stabilité des vins en bouteille. Enfin, l'acidité volatile, bien qu'à un niveau supérieur à celui enregistré de nos jours, reste acceptable pour l'époque.

L'eau de mer à pénétré lentement au travers du bouchon de liège; la teneur très élevée du sodium en atteste. Par ailleurs, l'augmentation de la concentration du milieu en sel a provoqué la précipitation d'une grande partie des composés phénoliques.

Ces différents résultats ne permettent pas, à eux seuls, de démontrer l'éventualité de l'élevage en barrique. Néanmoins, la présence d'une faible quantité d'acide ellagique indique que le vin a été en contact avec du bois ou des extraits de bois. Ce sont probablement des chênes européens ou des châtaigniers, qui possèdent des ellagitanins hydrosolubles s'hydrolysant facilement pour donner de l'acide ellagique.

\section{Mise en évidence de lignines dans le précipité phénolique dialysé}

Les chromatogrammes obtenus par CPG-SM (Fig. 1) à partir des thioacidolysats, après dérivation (triméthylsilylation), donnent relativement peu de pics. A partir des spectres de masse enregistrés en impact électronique, nous retrouvons deux structures provenant des motifs monométhoxylés (gaïacyles, $\mathrm{G}: \underline{\mathbf{1}}, \underline{\mathbf{3}}$ ) et deux provenant des motifs dimethoxylés (syringyles, $\mathrm{S}: \underline{\mathbf{2}}, \underline{\mathbf{4}}$ ) caractéristiques des lignines « $\mathrm{SG}$ » (formules Fig. 2). Si les produits $\underline{\mathbf{1}}$ et $\underline{\mathbf{2}}$ ont été parfaitement caractérisés à partir de produits purs [8], en revanche, l'attribution certaine des produits $\underline{\mathbf{3}}$ et $\underline{\mathbf{4}}$ reste basée seulement sur des modèles établis à partir de spectres de masse. Les travaux [2] suggèrent, toujours par spectrométrie de masse, qu'il s'agit de monomères obtenus par réarrangement des chaînes latérales en milieu acide $\left[\mathrm{CH}(\mathrm{SEt})_{2}-\mathrm{CHAr}-\mathrm{CH}_{2} \mathrm{SEt}\right]$. Cependant à ce jour, aucune preuve structurale (RMN) n'a formellement établi leur structure. Les spectres enregistrés pour ces molécules (Fig. 3) sont conformes a ceux enregistrés pour les lignines pures et conformes avec les données de la littérature [8]. Les produits $\underline{\mathbf{1}}$ et $\underline{\mathbf{2}}$ se présentent chacun sous forme de deux pics correspondant à leurs structures

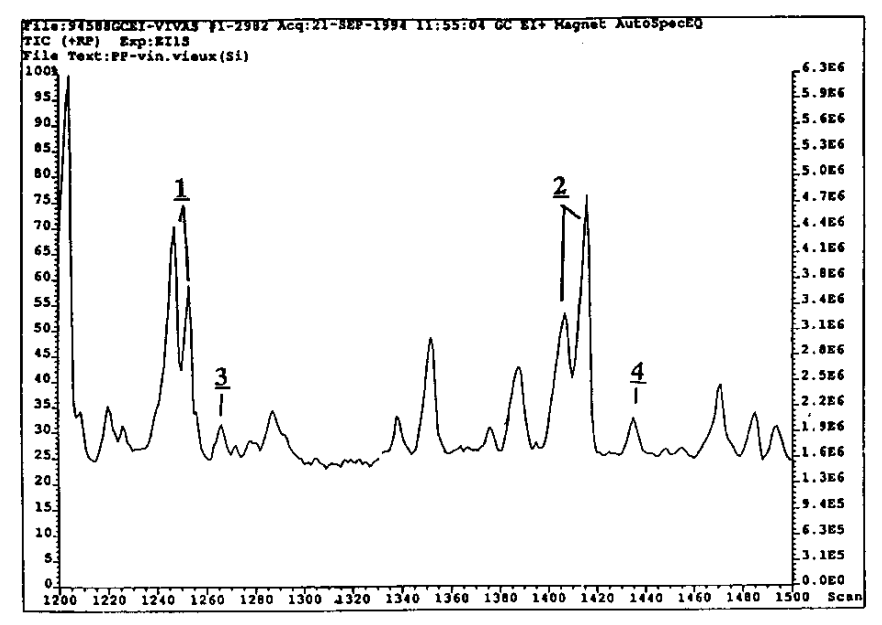

Fig. 1. Chromatogramme en phase gazeuse des produits triméthylsilylés provenant de la thioacidolyse du précipité dialysé du vin de 1868 (identification des pics cf. Fig. 2). 
érythro/thréo respectives. L'ensemble de ces molécules identifiées dans les produits de thioacidolyse des lignines<smiles>CCCC(CC)C(CC)c1ccc(O)c(OC)c1</smiles>

1<smiles>CCC(CC)C(CC)Cc1ccc(O)c(OC)c1</smiles>

$\underline{3}$<smiles>CCC(CC)C(CC)c1cc(OC)c(O)c(OC)c1</smiles>

2<smiles>CCC(CC)C(CC)Cc1cc(OC)c(O)c(OC)c1</smiles>

$\underline{4}$
Fig. 2. Principaux produits de thioacidolyse sous forme de dérivés thioéthylés ( 1 et 3 issus des motifs gaïacyles des lignines, 2 et 4 issus des motifs syringyles des lignines).
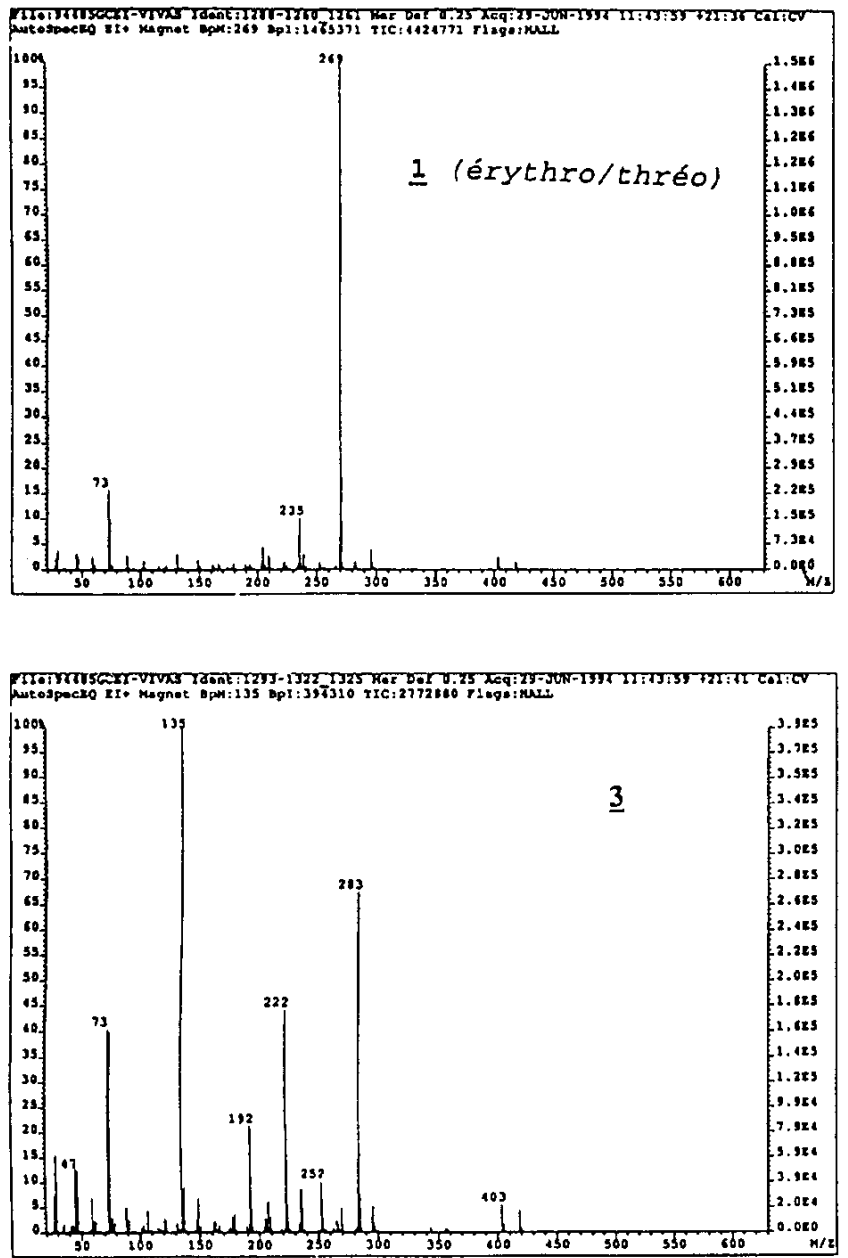

contenues dans le vin de 1868, sont typiques des liaisons « $\beta$-O-4 » présentes dans les lignines SG.

La présence de lignine de type SG spécifique des bois d'angiospermes (feuillus), dans le vin d'origine archéologique étudié ici, indique que celui-ci est resté en contact avec du bois pendant plusieurs mois. En effet, si les ellagitanins sont très rapidement solubilisés, en revanche, la dissolution de la lignine nécessite un long séjour en barriques et sa présence constitue donc la preuve d'une phase d'élevage de quelques mois.

\section{Conclusion}

Les analyses courantes pratiquées sur l'échantillon de 1868 montrent que celui-ci présente tous les indices d'un grand vin : niveau de maturité des raisins satisfaisant (degré alcoolique et acidité totale bons pour l'époque), conduite des vinifications dans de bonnes conditions (sucres réducteurs totaux inférieurs à $2 \mathrm{~g} / \mathrm{L}$, acidité volatile limitée et acide malique totalement dégradé). En outre, la richesse en composés phénoliques totaux et la teneur relativement élevée en procyanidines résiduelles, sont caractéristiques d'une cuvaison longue. Si ce n'est le caractère salé du vin, ses qualités gustatives sont tout à fait satisfaisantes au regard de son âge.
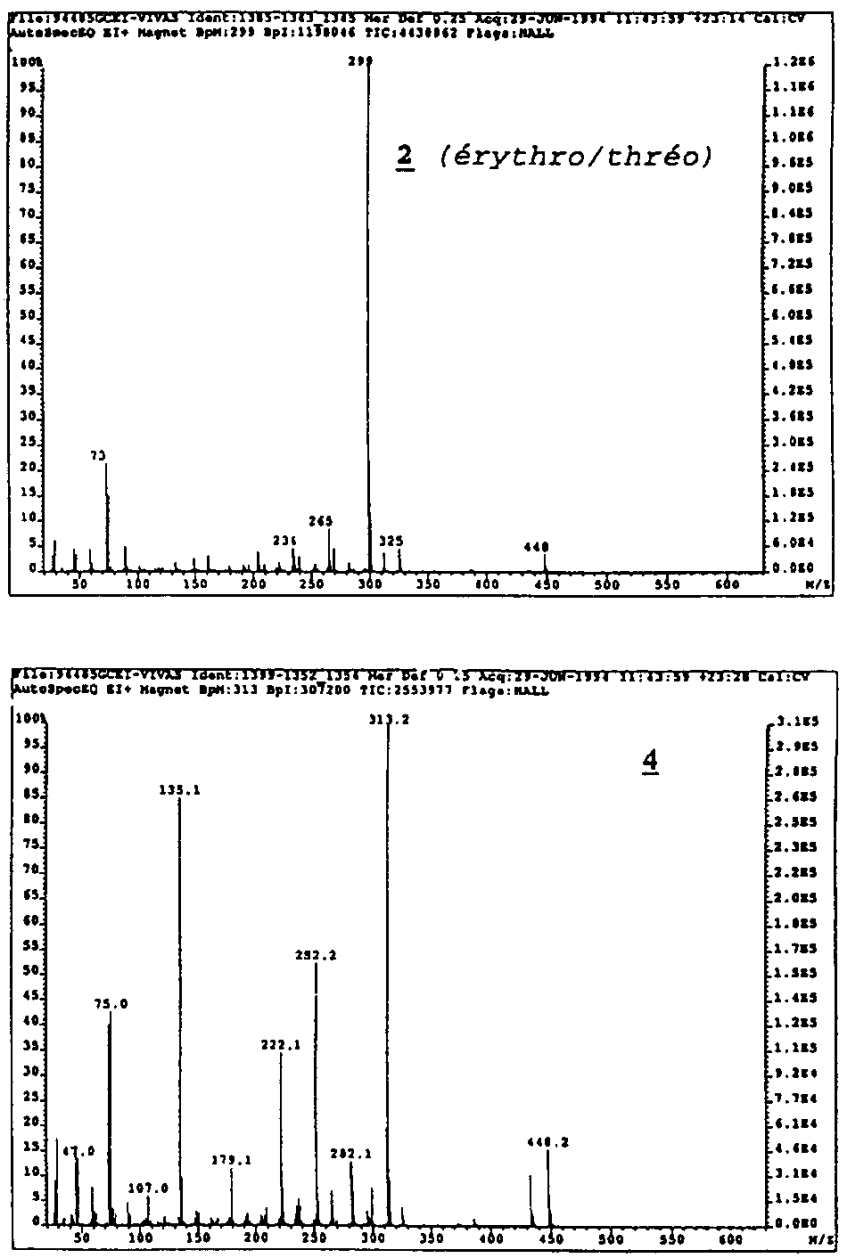

Fig. 3. Spectres de masse des différents produits identifiés dans le précipité dialysé du vin de 1868 (dérivés triméthylsilylés). 
La preuve d'une conservation prolongée du vin au contact de bois est donnée par l'identification des produits caractéristiques provenant de la thioacidolyse des lignines. La nature $\mathrm{SG}$ des lignines indique que le bois appartient à la famille des angiospermes et la présence d'acide ellagique libre tend a suggérer qu'il s'agit soit de chênes européens soit de châtaigniers.

Ainsi en 1868 pour de grands vins se présentant en bouteille pour la commercialisation, l'élevage en barriques sur plusieurs mois constituait donc une pratique vinicole effective. Alors qu'à cette époque les développements de l'Enologie Pastorienne ne s'étaient pas encore implantés dans le Bordelais [6], l'élevage constituait certainement, à cette époque plus qu'aujourd'hui, un risque réel pour la salubrité du vin. Néanmoins, les agréments apportés sur le plan gustatif ont probablement joué en faveur de cette pratique. Ce problème sanitaire ne se pose plus actuellement, l'œnologie moderne permettant des élevages en barriques avec des risques mieux contrôlés et des moyens de prévention bien mieux maîtrisés qu'en 1868.

\section{Remerciements}

Les auteurs remercient bien vivement les Établissements Cordier (Blanquefort), pour leur avoir fourni les échantillons archéologiques faisant l'objet de cette étude, ainsi que le Conseil Régional d'Aquitaine pour son appui logistique.

\section{Références}

1. Glories, Y. Recherches sur la matière colorante des vins rouges. Thèse doctorat en sciences, Université Bordeaux-1, 1978.
2. Hibino, T.; Shibata, D.; Ito, T.; Tsuchiya, D.; Higuchi, T.; Pollet, B.; Lapierre, C. Chemical properties of lignin from Aralia cordata. Phytochem. 1994, 37, 445-448.

3. Lapierre, C. Hétérogénéité des lignines de peuplier: Mise en évidence systématique. Thèse doctorat d'état, Université Parissud, 1986.

4. Médina, B. Application de la spectrométrie d'absorption atomique sans flamme au dosage de quelques métaux dans les vins. Thèse $3^{\text {ème }}$ cycle, Université de Bordeaux II, 1976.

5. O.I.V, Recueil des méthodes d'analyses des vins. O.I.V. (ed.), Paris, 1977.

6. Pasteur, L. Études sur le vin. $1^{\circ}$ édition, Librairie Savy, F., Paris, 1866.

7. Pepe, C.; Scribe, P.; Nallard, M. A.; Pollet, B.; Lapierre, C. Lignin signature in river suspension by capillary gas chromatography - mass spectrometry of thiocidolysis products. Analusis 1995, 23, 114-116.

8. Rolando, C.; Monties, B.; Lapierre, C. Thioacidolysis. In: «Methods in lignins chemistry», Lin, S. Y.; Dence, C. W. Eds., Springer-Verlag, Berlin, 1992; pp 334-349.

9. Salagoïty-Auguste, M. H.; Tricard, C.; Sudraud, P. Dosage simultané des aldéhydes, phénols et des coumarines par chromatographie liquide haute performance. Application aux vins et aux eaux-de-vie viellis en fûts de chêne. J. Chromatogr. 1987, 392, 379-387.

10. Taransaud, J. Le livre de la tonnellerie. La roue à livres diffusion Ed., Paris, 1976.

11. Viriot, C.; Scalbert, A.; Lapierre, C.; Moutounet, M. Ellagitannins and lignins in aging of spirits in oak barrels. $J$. Agric. Food Chem. 1993, 41, 1872-1879.

12. Vivas, N. La qualité du bois de chêne et son utilisation pour la vinification et l'élevage des vins. J. Sci. Tech. Tonnellerie 1995, 1, 1-16.

13. Vivas, N.; Glories, Y.; Bourgeois, G.; Vitry, C. Les ellagitanins de bois de cœur de différentes espèces de chênes (Quercus sp.) et de chataîgnier (Castanea sativa Mill.). Dosage dans les vins rouges élevés en barriques. J. Sci. Tech. Tonnellerie 1996, 2, 24-49.

14. Vivas, N.; Pianet, I.; Bourgeois, G.; Vitry, C.; Servens, C.; Glories, Y. Lignin fractions from Quercus robur L. and 